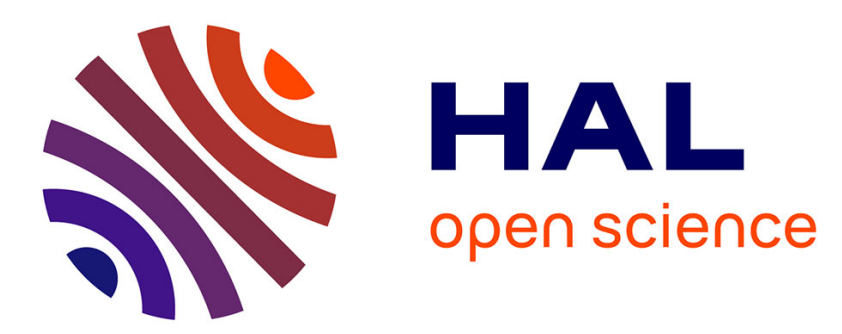

\title{
Synchronous and Asynchronous Evaluation of Dynamic Neural Fields
}

\author{
Nicolas P. Rougier, Axel Hutt
}

\section{To cite this version:}

Nicolas P. Rougier, Axel Hutt. Synchronous and Asynchronous Evaluation of Dynamic Neural Fields. Journal of Difference Equations and Applications, 2010, In press, In press (In press), 10.1080/10236190903051575 . inria-00403107

\section{HAL Id: inria-00403107 https://hal.inria.fr/inria-00403107}

Submitted on 9 Jul 2009

HAL is a multi-disciplinary open access archive for the deposit and dissemination of scientific research documents, whether they are published or not. The documents may come from teaching and research institutions in France or abroad, or from public or private research centers.
L'archive ouverte pluridisciplinaire HAL, est destinée au dépôt et à la diffusion de documents scientifiques de niveau recherche, publiés ou non, émanant des établissements d'enseignement et de recherche français ou étrangers, des laboratoires publics ou privés. 
RESEARCH ARTICLE

\title{
Synchronous and Asynchronous Evaluation of Dynamic Neural Fields
}

\author{
N.P. Rougier* \& A. Hutt \\ LORIA - Campus Scientifique - BP 239 - 54506 Vandoeuvre-les-Nancy Cedex \\ (Received 00 Month 200x; in final form 00 Month 200x)
}

\begin{abstract}
In [26], we've introduced a dynamic model of visual attention based on the Continuum Neural Field Theory [29] that explained attention as being an emergent property of a dynamic neural field. The fundamental property of the model is its facility to select a single stimulus out of several perfectly identical input stimuli by applying asynchronous computation. In the absence of external noise and with a zero initial state, the theoretical mathematical solution of the field equation predicts the final equilibrium state to equally represent all of the input stimuli. This finding is valid for synchronous numerical computation of the system dynamics where elements of the spatial field are computed all together at each time point. However, asynchronous computation, where elements of the spatial field are iterated in time one after the other yields different results leading the field to move towards a single stable input pattern. This behavior is in fact quite similar to the effect of noise on dynamic fields. The present work aims at studying this phenomenom in some details and characterizes the relation between noise, synchronous evaluation (the "regular" mathematical integration) and asynchronous evaluation in the case of a simple dual particle system. More generally, we aim at explaining the behavior of a general differential equation system when it is considered as a set of particles that may or may not iterated by synchronous computations.
\end{abstract}

Keywords: Synchronous computation, Asynchronous computation, Local update, Dynamic Neural Fields

AMS Subject Classification: PACS: 02.30.Hq, 07.05.Mh, 84.35.+i

\section{Introduction}

Most computational paradigms linked to artificial neural networks (using rate code) or cellular automata use implicitly what is called synchronous evaluation of activity. This means that information at time $t+d t$ is evaluated exclusively on information available at time $t$. The explicit numerical procedure of performing such a synchronized update is to implement a temporary buffer at the unit level where activity computed at time $t+\Delta t$ is stored. Once all units have evaluated their activity at time $t+\Delta t$, the current activity is replaced by the content of the buffer. We point out that other update procedures have been developed [22] but the basic idea remains the same, namely not to mix information between time $t$ and time $t+\Delta t$. To perform such a synchronization, there is thus a need for a global signal that basically tell units that evaluation is over and they can replace their previous activity with the newly computed one. At the computational level, this synchronization is rather expensive and is mostly justified by the difficulty of handling asynchronous models. For example, cellular automata have been extensively studied during the past decades for the synchronous case and mathematical

${ }^{*}$ Corresponding author. Email: Nicolas.Rougier@loria.fr 
studies have been performed. However, recent theoretical works on asynchronous computation in distributed computational networks [3, 4] and cellular automata [14] showed that the behavior of these same models and associated properties may be of a radical different nature depending on the level of synchrony of the model (you can asynchronously evaluate only a subpart of all the available automata). In the framework of computational neuroscience we may then wonder what is the relevance of synchronous evaluation since most generally, the system of equations is supposed to give account of a population of neurons that have no reason to be synchronized (if they are not provided with an explicit synchronization signal). We would like in this article to shed some light on such phenomenom and the consequences on modelling, especially in the framework of dynamic neural fields. After defining what we call synchronous and asynchronous evaluation of a system of differential equation, we introduced some results relative to dynamic neural field that underline clearly (and numerically) the difference between synchronous and asynchronous evaulation. To study this phenomenon, we then consider a degenerated system made of only two potentials that will help us to understand what is going on. Finally, we make a conjecture regarding the link between synchronous and asynchronous evaluation.

\section{Synchronous and Asynchronous Evaluation}

In order to define what we called asynchronous evaluation of a differential system, we need first to define properly synchronous evaluation. Let us consider a generic discrete set of $n$ first order differential equations:

$$
\begin{array}{r}
\forall i \in[1, n], x_{i}: \mathbb{R}^{+} \rightarrow \mathbb{R} \\
\frac{d x_{i}(t)}{d t}=f_{i}\left(x_{1}(t), \ldots, x_{n}(t)\right)
\end{array}
$$

with a set of initial conditions:

$$
\left[x_{1}(0), \ldots, x_{n}(0)\right] \in \mathbb{R}^{n}
$$

When symbolic resolution is not possible, one can approximate the evolution of such a system using numerical integration, i.e. low-order methods as the Euler-forward or methods of higher order such as the Runge-Kutta methods [32]. For sake of notation, we will use the Euler-forward method in the following but the same definitions apply to other methods as well.

The Euler method provides us with an approximation for first order differential equations using the approximation

$$
\Delta x_{i}(t)=\Delta t f_{i}\left(x_{1}(t), \ldots, x_{n}(t)\right)
$$

$$
\begin{aligned}
\Delta x_{i}(t) & =\Delta t f_{i}\left(x_{1}(t), \ldots, x_{n}(t)\right), i \in \mathcal{S} \\
\Delta x_{j}(t) & =0, j \in \overline{\mathcal{S}}
\end{aligned}
$$


where $\mathcal{S}$ is a set of integers between 1 and $n$ and $\overline{\mathcal{S}}$ represents its complement. Interestingly, Eq. (4) reveals that the systems fixed points are independent from the choice of $\mathcal{S}$ since $\Delta x_{i}(t)=0$ stipulates $f_{i}\left(x_{1}(t), \ldots, x_{n}(t)\right)=0$.

The following paragraphs distinguish different choices of the set $\mathcal{S}$ yielding different evaluation types.

\subsection{Synchronous evaluation}

The conventional update rule evaluates all elements synchronously, i.e. $\mathcal{S}$ is the set of all integers between 1 and $n$, i.e. $\mathcal{S}=\{1, \ldots, n\}$. Consequently (5) read

$$
x_{i}(t+\Delta t)=x_{i}(t)+\Delta t f_{i}\left(x_{1}(t), \ldots, x_{n}(t)\right), \forall i=1, \ldots, n
$$

This approximation is most commonly iterated over time until the desired state is reached, e.g. a given final time $t_{\text {final }}$. The pseudo-code for this computation type reads

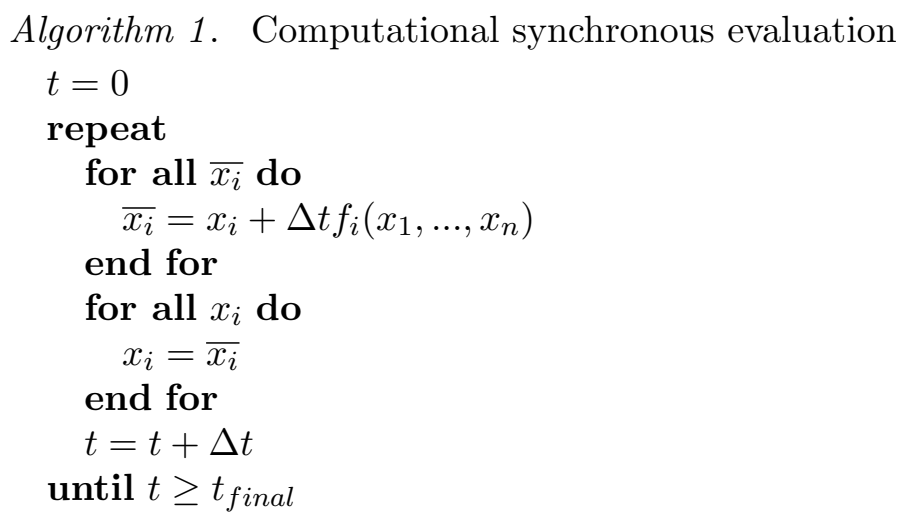

This algorithm computes $n$ updates in each time interval $\Delta t$.

From a mathematical perspective, this is what corresponds to the conventional definition of the Euler-forward approximation. From a more physical perspective, this also makes sense if we consider $t$ as the common or unified time for all the different variables $x_{i}(t)$.

We point out that the evaluation scheme (6) is a multi-dimensional map of the type $\mathbf{x}_{i+1}=\mathbf{g}\left(\mathbf{x}_{i}\right)$ with vectors $\mathbf{x}_{i}, \mathbf{g} \in \Re^{n}$ and obeys the mathematical rules of differential equations for $\Delta t \rightarrow 0$.

\subsection{Asynchronous evaluation}

However, as we underlined in the introduction, this unification of time is not that straightforward if we consider those equations to represent neuron potentials that can now be considered largely as indepedent biological elements, even if they are linked to other neurons, e.g. through synapses. Consequently, each element might have its own time and hence its own update time. To give a mathematical formulation of this situation, the set $\mathcal{S}$ in $(5)$ is chosen to $\mathcal{S}=\operatorname{rand}(n)$ containing the single integer chosen randomly from the interval $[1 ; n]$. Hence each element $x_{i}$ is updated separately and the evaluation is asynchronous. This evaluation procedure is also called local update $[9,12,23]$. In other words at each time point the asynchronous procedure updates a single element $i$ only and this element is chosen randomly. 
In mathematical terms, the numerical evaluation scheme can be formulated by

$$
x_{i}(t+\Delta t / n)=x_{i}(t)+\delta_{i j} \xi(j)(\Delta t / n) f_{i}\left(x_{1}(t), \ldots, x_{n}(t)\right), \forall i=1, \ldots, n .
$$

The term $\xi(j) \in[1 ; n]$ represents a random process, which fills the interval $[1 ; n]$ with integers in random order. If the interval is filled, the interval is emptied and the filling process starts again. This process is used in physical chemistry and biology and is known as random sequential adsorption, see e.g. [8]. We conclude here that by virtue of the random nature of the update rule, the asynchronously updated systems do not obey the mathematical rules of differential equations and hence novel effects may occur. However we will see in the following sections that the limit $\Delta t \rightarrow 0$ diminishes the random effects and the dynamics obtained by asynchronous evaluation approach the synchronous results, i.e. the analytical results gained for differential equations.

Two different ways to implement such an asynchronous procedure are given in the following algorithms, which ensure $n$ computations in each interval $\Delta t$.

Algorithm 2. Computational asynchronous evaluation (non-uniform)

$$
\begin{aligned}
& t=0 \\
& \text { repeat } \\
& \quad i=\operatorname{rand}(n) \\
& \quad x_{i}=x_{i}+\Delta t f_{i}\left(x_{1}, \ldots, x_{n}\right) \\
& \quad t=t+\Delta t / n \\
& \text { until } t \geq t_{\text {final }}
\end{aligned}
$$

Here $\operatorname{rand}(n)$ denotes a random integer taken from the interval $[1 ; n]$. In each time interval $\Delta t / n$, we update only one $x_{i}(t)$. In statistical terms, this evaluation resembles draws in an urn model with return while $n$ elements are drawn from the urn in each time interval.

We may also define a more uniform asynchronous evaluation which guarantees that each of the $x_{i}$ is evaluated only once in the time interval:

Algorithm 3. Computational asynchronous evaluation (uniform)

$$
\begin{aligned}
& t=0 \\
& \text { repeat } \\
& \quad \text { index }=\text { shuffle }([1 . . n]) \\
& \quad \text { for } i=1 \text { to } n \text { do } \\
& \quad x_{\text {index }[i]}=x_{\text {index }[i]}+\Delta t f_{\text {index }[i]}\left(x_{1}, \ldots, x_{n}\right) \\
& \quad \text { end for } \\
& \quad t=t+\Delta t \\
& \text { until } t \geq t_{\text {final }}
\end{aligned}
$$

Here, shuffle([1..n]) denotes the sequence randomization of integers in the interval $[1 ; n]$. This evaluation scheme corresponds to an homogenous system where all the $x_{i}$ evolve along a common time axis. In a statistical sense, this evaluation resembles the urn model without return and a complete return of all elements after the time $\Delta t$.

In addition we mention that the asynchronous evaluation scheme is not restricted to explicit evaluation schemes such as the Euler method and may be formulated for semi-implicit and implicit scheme as well.

The natural question concerning the differences between synchronous and asynchronous evaluation is to know whether they approximate the same system or if 
they are different in nature. To do so, we would like first to illustrate these two evaluation types using a model of dynamic neural field.

\section{Dynamic Neural Fields}

Biological neural networks exhibit multiple spatial and temporal scales and thus it is a difficult task to model their spatio-temporal dynamics in all scales. Nevertheless to explain various phenomena found experimentally, previous studies have focussed on specific spatial and temporal scales. A well-studied description level is the neural population level which considers the population firing rate of the neural ensemble, the spatial scale of hundreds of micrometers and the temporal scale of few milliseconds. This model type, called neural field, allowed for the mathematical description of experimental phenomena, such as visual hallucinations [13], spiral waves in the cortex [18], the power spectrum in anesthesia [6, 28] and sleep cycles [27]. Moreover neural fields are supposed to model the storage of patterns in neural populations, such as breathers [15] or static bumps [21, 25]. Such phenomena are self-stabilizing in the absence of external stimuli, while some recent studies investigated the effect of external inputs on waves [16] and static localized activity [31].

We have been studying the Continuum Neural Field Theory (CNFT) $[1,2,10,20$, $29,33,34]$ extensively in [26] where we have introduced a dynamic model of visual attention that explains attention as being an emergent property of such dynamic neural field. The fundamental property of the model is its facility to select a single stimulus out of several perfectly identical input stimuli at the presence of spatial input noise. In other words, the model is able to make a choice by selecting an input among those available. Moreover the previous study [26] considers asynchronous numerical computation.

However in the absence of spatial input noise, the mathematical solution of the field equation predicts the final equilibrium state to equally represent all of the input stimuli. The reason for the selection to occur as shown in [26] is indeed the asynchronous evaluation that introduces the neccessary asymmetry that lead the system to reach an equilibrium state reflecting just a single input stimulus. Moreover, we point out that this selection can not be predicted by neural field theory, since asynchronous evaluation implies a random process and thus does not obey the analysis rules of differential equations.

The following paragraphs illustrate these results in the CNFT for synchronous and asynchronous evaluation, two different inputs and various time intervals $\Delta t$.

\subsection{Continuum Neural Field Theory}

Using notations introduced by [1], a neural position is labelled by a vector $\mathbf{x}$ on a manifold $\mathcal{M}$. The field variable represents the membrane potential of a neuronal population at the point $\mathbf{x}$ at time $t$ and is denoted by $u(\mathbf{x}, \mathbf{t})$. It is assumed that there are lateral connections with the weight function $w_{M}\left(\mathbf{x}-\mathbf{x}^{\prime}\right)$ which is in our case a difference of Gaussian function as a function of the distance $\left|\mathbf{x}-\mathbf{x}^{\prime}\right|$. The model also considers an afferent connection weight function $s(\mathbf{x}, \mathbf{y})$ from the position $\mathbf{y}$ in the manifold $M^{\prime}$ to the point $\mathbf{x}$ in $M$. This function weights the input into the spatial field under study and thus reflects receptive field connections. The 
membrane potential $u(\mathbf{x}, t)$ satisfies the following equation (8):

$$
\begin{aligned}
\tau \frac{\partial u(\mathbf{x}, t)}{\partial t}=-u(\mathbf{x}, t) & +\int_{M} w_{M}\left(\mathbf{x}-\mathbf{x}^{\prime}\right) f\left[u\left(\mathbf{x}^{\prime}, t\right)\right] d \mathbf{x}^{\prime} \\
& +\int_{M^{\prime}} s(\mathbf{x}, \mathbf{y}) I(\mathbf{y}, t) d \mathbf{y}+h
\end{aligned}
$$

where $\tau$ denotes the synaptic time constant, $f$ represents the mean firing rate as the function of the membrane potential $u$ of the population, $I(\mathbf{y}, t)$ is the input from position $\mathbf{y}$ at time $t$ in $M^{\prime}$ and $h$ is the mean neuron threshold. In detail, the firing rate function $f$ is chosen as the piece-wise linear function

$$
f[u]= \begin{cases}0 & \text { if } u \leq 0 \\ u & \text { if } 0<u<1 \\ 1 & \text { if } u \geq 1\end{cases}
$$

the lateral connctivity function $w_{M}$ reads

$$
w_{M}\left(\mathbf{x}-\mathbf{x}^{\prime}\right)=A e^{\frac{\left|\mathbf{x}-\mathbf{x}^{\prime}\right|^{2}}{a^{2}}}-B e^{\frac{\left|\mathbf{x}-\mathbf{x}^{\prime}\right|^{2}}{b^{2}}} \text { with } A, B, a, b \in \Re^{*+}
$$

and the afferent connections are described by

$$
s(\mathbf{x}, \mathbf{y})=C e^{\frac{|\mathbf{x}-\mathbf{y}|^{2}}{c^{2}}} \text { with } C, c \in \Re^{*+}
$$

In the following, the spatial domain is $[-0.5,0.5]^{2}$ on both manifold $\mathcal{M}, M^{\prime}$ involving periodic boundary conditions.

\subsection{Symmetric input}

We consider the case where there are two distinct gaussian inputs within the $\mathcal{M}$ manifold, one centered at $\left(\frac{1}{3}, \frac{1}{3}\right)$ and one centered at $\left(-\frac{1}{3},-\frac{1}{3}\right)$ such that:

$$
\begin{array}{r}
G(x, y, \sigma)=e^{-\frac{x^{2}+y^{2}}{2 \sigma^{2}}} \\
\mathcal{M}(x, y)=G\left(x-\frac{1}{3}, y-\frac{1}{3}, 1\right)+G\left(x+\frac{1}{3}, y+\frac{1}{3}, .1\right)
\end{array}
$$

The manifolds $\mathcal{M}$ and $\mathcal{M}^{\prime}$ have been respectively discretized into a set of $30 \times 30$ units and each of the unit of $\mathcal{M}^{\prime}$ receives the corresponding input from $\mathcal{M}$ (function $s$ from Eq. (11) is degenerated into a single afferent point).

Starting from a perfectly null state in the output and using equations introduced in the previous section and synchronous evalutation (see algo. 1), we ran simulation for 10 seconds using $\Delta t=1000 \mathrm{~ms}$ and $\Delta t=10 \mathrm{~ms}$ (see figure 1). As predicted, resulting output patterns represent both input stimuli. Aynchronous evalutation (see algo. 3) yields different results. For a large $\Delta t=1000 \mathrm{~ms}$, only one of the input is fully represented in the output while the other vanished (see fig. 2). To make sure that one bump survives only, we examined numerically the neural field activity at the location of the expected second bump and found vanishing activity. This result indicates that the second bump vanished indeed. 

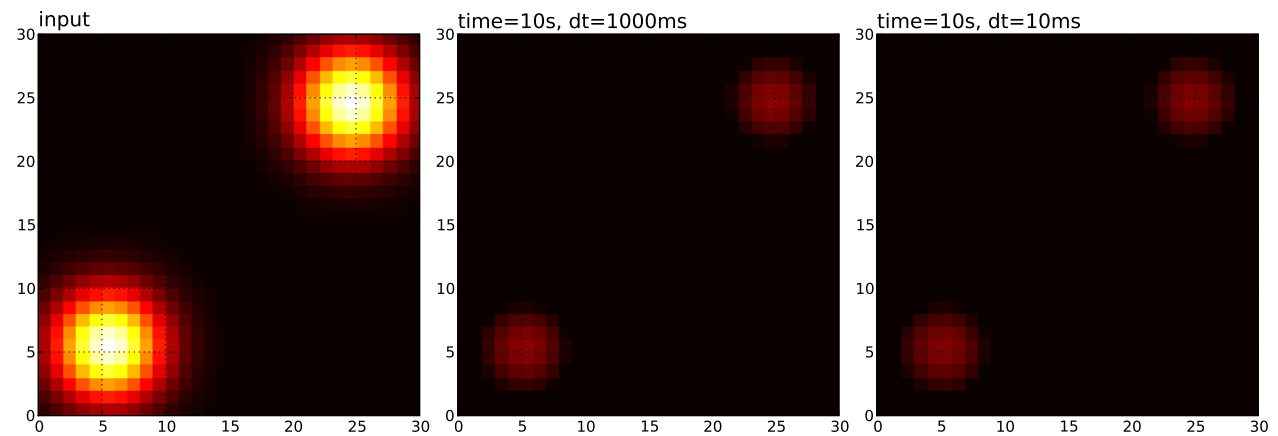

Figure 1. Symetric input, synchronous evaluation
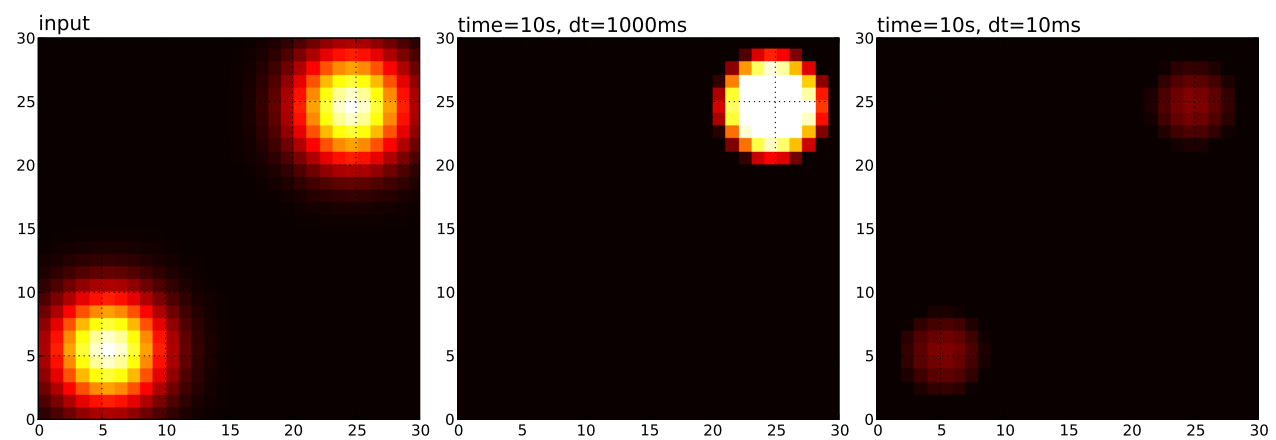

Figure 2. Symetric input, uniform asynchronous evaluation

Only when we reduce $\Delta t$ to $10 \mathrm{~ms}$, we then observe results comparable to the synchronous case. Note that we've also tested algorithm 2 (not represented) and obtained the same results.

At a first glance, the disappearence of one bump and thus the symmetry breaking with $\Delta t=1000 \mathrm{~ms}$ in Fig. 2 is surprising and can not be understood by neural field theory. Since the two bumps re-occur for the smaller time step $\Delta t=10 \mathrm{~ms}$, we argue that the disappearence of one bump results from the asynchronous evaluation scheme, which implies random processes (section2.2) and hence can not be understood by mathematical analysis based on the Eq. (8).

\subsection{Asymmetric input}

We also consider asymetric input where input is given by:

$$
\begin{array}{r}
G(x, y, \sigma)=e^{-\frac{x^{2}+y^{2}}{2 \sigma^{2}}} \\
\mathcal{M}(x, y)=\frac{1}{2} G\left(x-\frac{1}{3}, y-\frac{1}{3}, .1\right)+G\left(x+\frac{1}{3}, y+\frac{1}{3}, .1\right)
\end{array}
$$

and ran simulations as in a similar way as of the previous subsection. Since the input is not symetric anymore, we observe in the output that the most salient stimulus is fully represented (see fig. 3 ).

In the case of asynchronous evaluation (either algo. 2 or 3 ), we obtained exactly the same results (see fig. 4 ), whatever the $\Delta t$. This lead us to consider the nature of the final states and to make the link between stability of the state and the probability to reach such a state in case of asynchronous evaluation. Since the CNFT may be too complex for a thorough analysis, we considered a reduced model to explain the underlying dynamics. 

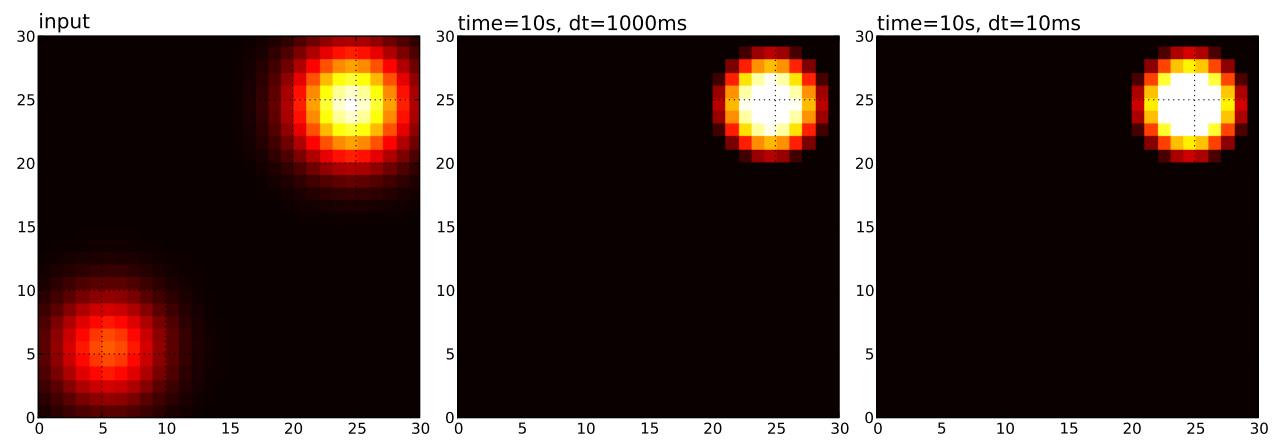

Figure 3. Asymmetric input, synchronous evaluation
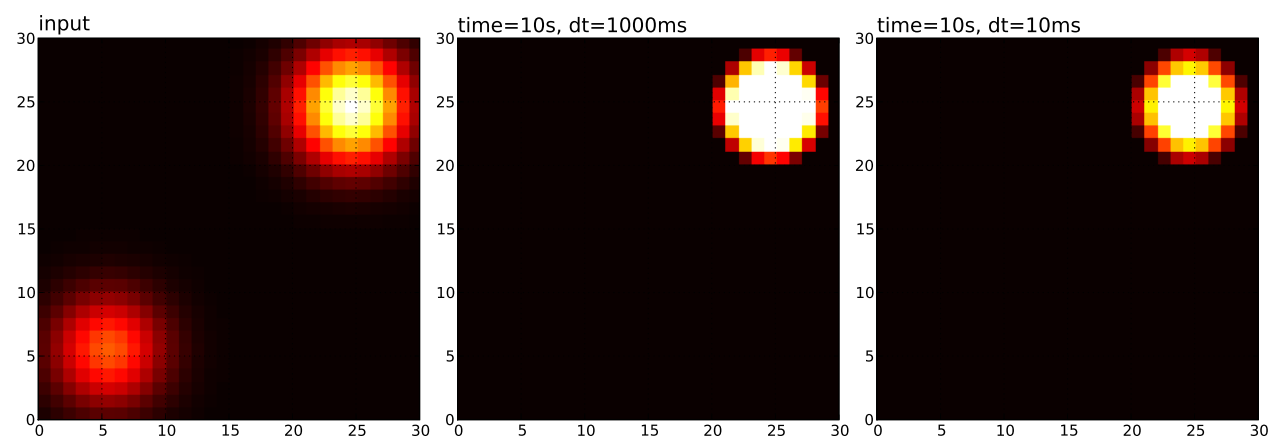

Figure 4. Asymmetric input, uniform asynchronous evaluation

\section{The reduced model}

To explain in detail the spatio-temporal behavior in section 3, we introduce a lowdimensional model, whose behavior reflects the major phenomena observed in the CNFT. The model equations read

$$
\begin{aligned}
& \dot{y}=-\alpha y+(y-z)(1-y)+\alpha I_{y} \\
& \dot{z}=-\alpha z+(z-y)(1-z)+\alpha I_{z}
\end{aligned}
$$

with the absorbing boundary conditions $y\left(t_{0}\right)=0 \rightarrow y\left(t>t_{0}\right)=0, y\left(t_{0}\right)=1 \rightarrow$ $y\left(t>t_{0}\right)=1, z\left(t_{0}\right)=0 \rightarrow z\left(t>t_{0}\right)=0, z\left(t_{0}\right)=1 \rightarrow z\left(t>t_{0}\right)=1$. Here $I_{y}, I_{z}$ are the external inputs which are specified to $I_{y}=1, I_{z}=I$ in the following discussion. Further we choose $0<\alpha<2$ and the parameter $I$ is the constant external input with $0<I \leq 1$.

Since dynamic neural fields are mainly concerned with competition among units, we build this model in order to benefit from a very simple competition mechanism where the growing of one variable is conditionned to both its difference from the other variable and to how far it is from the input. For example, if at a given time $y$ is greater than $z$, then the term $y-z$ is positive and lead $y$ to reach the input value $I_{y}$. At the same time, the variable $z$ tends to decrease since the term $z-y$ is now negative. The greater this difference is, the faster the two variables will reach their respective final state. If at any time the two variables are equal, then they do not influence each other and can reach their respective inputs.

Although no direct derivation of the model (14) from the neural field equations (8) exist, we may relate parameters of both models. For instance the parameter $\alpha$ in (14) defines the susceptibility of the system to the external input and reflects the rate of convergence to fixed points, i.e. its stability. This can be seen at $(x=0, y=$ 

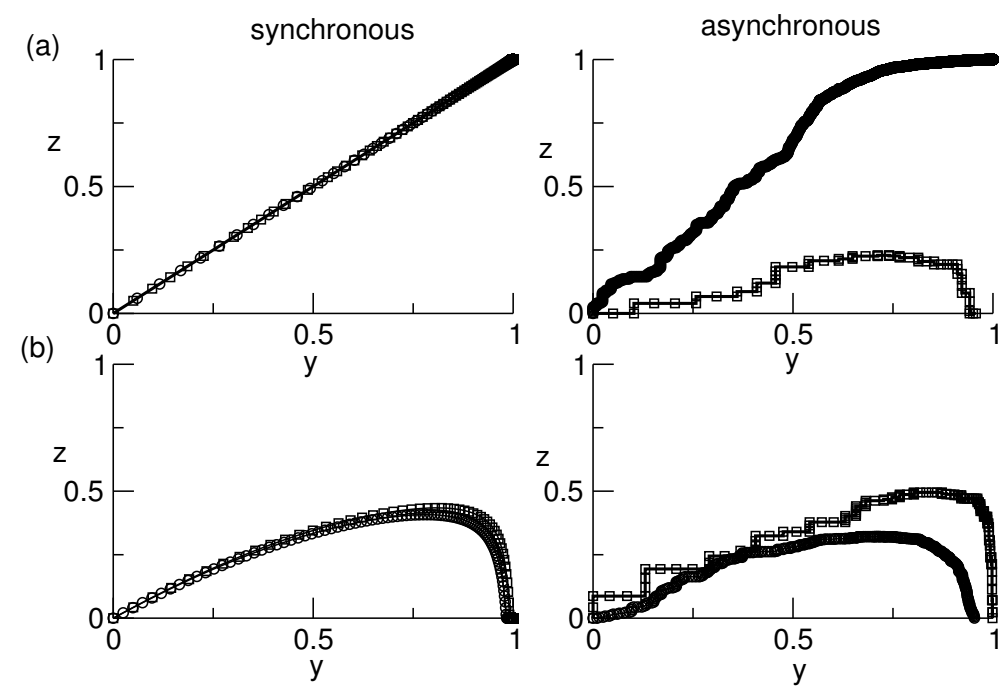

Figure 5. Example trajectories based on the reduced model for the synchronous and the uniform asynchronous evaluation, two different inputs $I$ and two values of $\Delta t$. (a) $I=1$, (b) $I=0.85$. The values of $\Delta t$ are chosen to $\Delta t=0.1$ (squares) and $\Delta t=0.01$ (circles). Further $\alpha=0.5$ and the initial conditions are $x(0)=y(0)=0$.

$0)$ where $(\dot{y}, \dot{z})=\alpha\left(I_{y}, I_{z}\right)$ and at $(x=0, y=0)$ where we find $(\dot{y}, \dot{z})=-\alpha(y, z)$. In the neural field model it is well-known that the nonlinear gain, i.e. the steepness of the transfer function, defines the excitability of the system, i.e. the susceptibility to external input, and the stability of the field [7, 19, 30]. Hence, the steepness of the transfer function, i.e. the mean firing rate function, in (8) and $\alpha$ in (14) are strongly related.

Figure 5 presents some numerical solutions of (14). In the case of a symmetric input, i.e. $I=1$, the synchronous evaluation yields the final state $(1,1)$ for both values of $\Delta t$ (Fig. 5(a), left panel). Consequently the input $(1,1)$ yields the equilibrium $(1,1)$ and thus resembles the CNFT-result shown in Fig. 1. Applying the uniform asynchronous evaluation scheme introduced in section 2.2 the system reaches the state $(1,1)$ for small $\Delta t$, but approaches the state $(1,0)$ for large $\Delta t$, cf. Fig. (5)(a), right panel. This behavior shows good accordance to the corresponding CNFT-case observed in Fig. 2. Moreover considering the different input stimulus $I<1$ (Fig. 5(b)), the synchronous and asynchronous computation yield the same final stationary state irrespective the value of $\Delta t$. This result also shows good accordance to the findings in the CNFT-model, cf. Figs. (3), (4). Summarizing, the low-dimensional model (14) shares the major dynamical properties of the CNFTmodel (8) and replaces it in good approximation. Consequently the detailed study of the low-dimensional model allows for deeper insight into the understanding of the CNFT-model.

\subsection{Dynamical properties}

To better understand the dynamical behavior observed in Fig. 5, let us study to the stationary states of the model (14) subjected to the external input $I$. We find a critical input $I_{c}=1-\alpha / 4$, which allows to distinct two cases for $0<y<1,0<$ $z<1$ :

- for $0<I<I_{c}$ a single fixed point FP exists at

$$
y_{0}=(I+1) / 2-\alpha, z_{0}=(I+1) / 2
$$


(a)

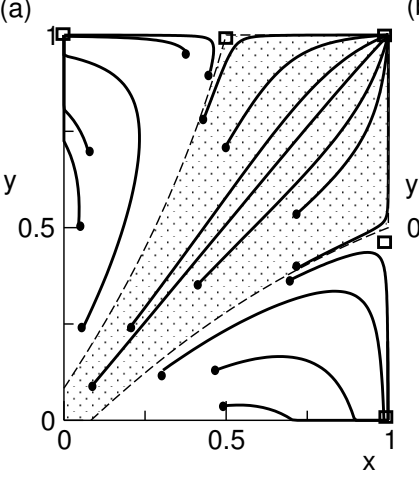

(b)

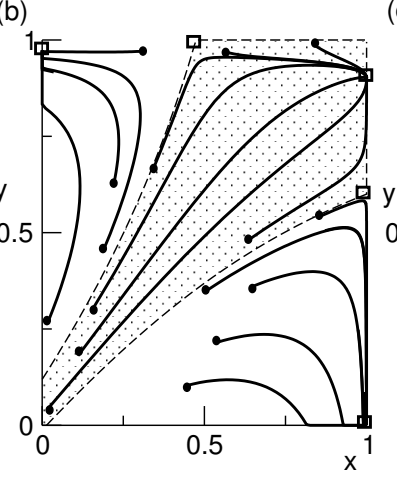

(c)

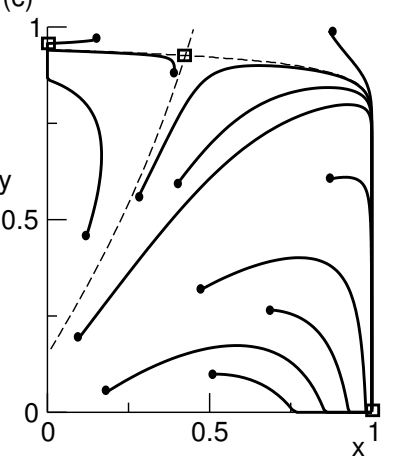

Figure 6. The topography of the low-dimensional model (14) for different inputs $I$. (a) $I=1>I_{c}$, (b) $I=0.92>I_{c}$ and (c) $I=0.85<I_{c}$. Here $\alpha=0.5$ which leads to $I_{c}=0.845$. The solid lines in the panels represent the trajectories with initial points denoted by filled dots. Further the dashed lines represent the separatrix, the dotted domain in (a) and (b) denote the basin of attraction of FP1 and the open squares mark the positions of fixed points calculated analytically.

- for $I_{c} \leq I \leq 1$ three fixed points exist at

$$
\begin{aligned}
& F P 1: y_{0}=1 \quad, \quad z_{0}=(\alpha-2)\left(1+\sqrt{1+4(\alpha I-1) /(\alpha-2)^{2}}\right) / 2 \\
& F P 2: y_{0}=1 \quad, \quad z_{0}=(\alpha-2)\left(1-\sqrt{1+4(\alpha I-1) /(\alpha-2)^{2}}\right) / 2 \\
& F P 3: y_{0}=(I+1) / 2-\alpha \quad, \quad z_{0}=(I+1) / 2 .
\end{aligned}
$$

In the specific case $I=1$, the fixed points read

$$
\begin{aligned}
& F P 1: y_{0}=1 \quad, \quad z_{0}=1 \\
& F P 2: y_{0}=1 \quad, \quad z_{0}=1-\alpha \\
& F P 3: y_{0}=1-\alpha \quad, \quad z_{0}=1 .
\end{aligned}
$$

To gain the linear stability conditions of the corresponding fixed points, we linearize Eqs. (14) about the corresponding fixed points and find two real-valued Lyapunov exponents $\lambda_{1}, \lambda_{2}$ for each fixed point:

- for $0<I<I_{c}$, the single fixed point FP is a saddle node with $\lambda_{1}<0, \lambda_{2}>0$.

- for $I_{c} \leq I \leq 1, \mathrm{FP} 1$ is a stable node and FP2 and FP3 are saddle nodes.

Moreover, the system evolves on the boundary and a linear stability analysis reveals fixed points

$$
\begin{aligned}
& F P B y: y_{0}=(1-\alpha)\left(1+\sqrt{1+4 \alpha I /(1-\alpha)^{2}}\right) / 2 \\
& F P B z: x_{0}=(1-\alpha)\left(1+\sqrt{1+4 \alpha /(1-\alpha)^{2}}\right) / 2,
\end{aligned}
$$

which are stable irrespective to the choice of $0<I<1$.

Figure 6 summarizes the latter analytical results and reveals a basin of attraction of FP1 for $I_{c} \leq I \leq 1$ which vanishes for smaller values $I<I_{c}$. In general we observe that $1>I$, i.e. $I_{y}>I_{z}$ and the input into $y$ is stronger than into $z$, yields an increase of the basin of attraction of FPBy. 


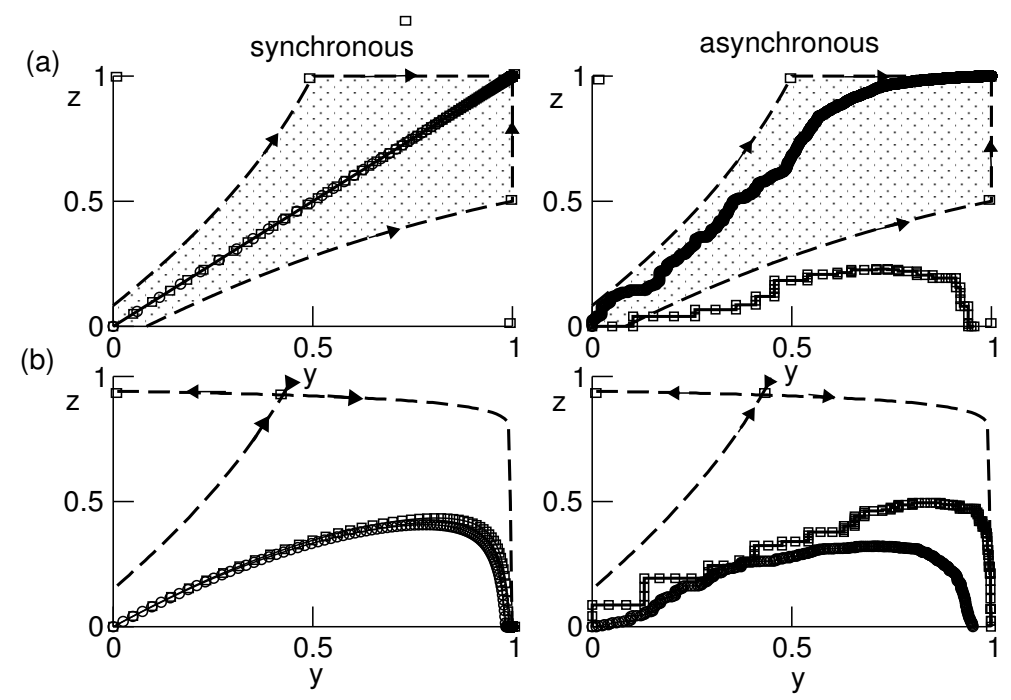

Figure 7. Trajectories and topology overlayed in single plots. (a) $I=1>I_{c}$, (b) $I=0.85<I_{c}$. Other parameters and symbols are taken from Figs. 5 and 6.

(a)

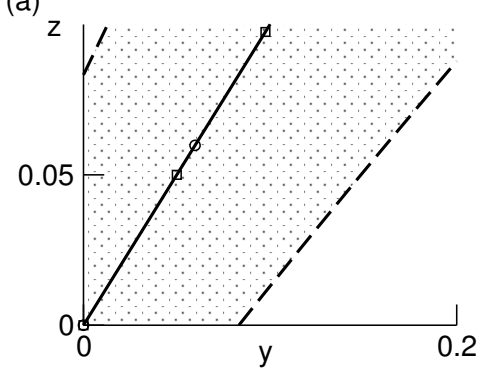

(b)

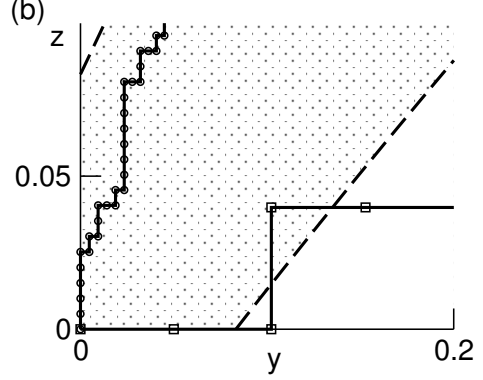

Figure 8. Focus on panels in Fig. 7(a). (a) synchronous computation, (b) asynchronous computation. Circles and squares encode $\Delta t=0.01$ and $\Delta t=0.1$.

\subsection{Effect of the external input}

To further investigate the systems evolution for different inputs, Fig. 7 overlays the trajectories from Fig. 5 and the systems topology shown in Fig. 6. For $I=1$, we observe that the trajectories computed synchronously start in the basin of attraction of FP1 and stay there until they reach $(1,1)$ (Fig. 7(a), left panel), while trajectories computed asynchronously my leave the basin of attraction for large $\Delta t$, see Fig. 7(a), right panel. Moreover, $I=0.85$ destroys FP1 and its basin of attraction and puts the initial point $(0,0)$ into the basin of attraction of $(1,0)$, (Fig. $7(\mathrm{~b}))$. Consequently all trajectories shown approach the stationary point $(1,0)$. In general decreasing $I$ diminishes the input into $z$ and increases the basin of attraction of $(1,0)$. This behavior resembles the results in neural fields for large enough $\Delta t$, where the stronger input is prefered.

In addition we observe that $I=1$ allows the trajectories to approach the final states $(1,0),(0,1)$ and $(1,1)$, while $I=0.85<I_{c}$ yields either $(1,0)$ or $(0,1)$. Hence input stimuli $I_{c}<I=I_{z}<1$ are different from $I_{y}=1$, but may not be detected as different since the systems trajectory may approach $(1,1)$. In turn the larger $I_{c}$, the better the system can distinguish different stimuli $I_{y}$ and $I_{z}$.

\subsection{Effect of the computation type and $\Delta t$}

To understand the different effects of synchronous and asynchronous computation, Fig. 8 presents a focus of the panels in Fig. 7(a). In the case of synchronous com- 
(a)

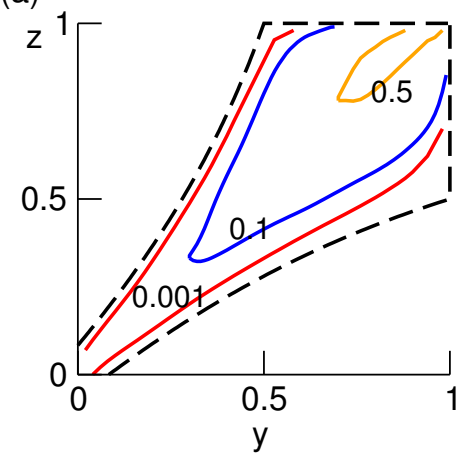

(b)

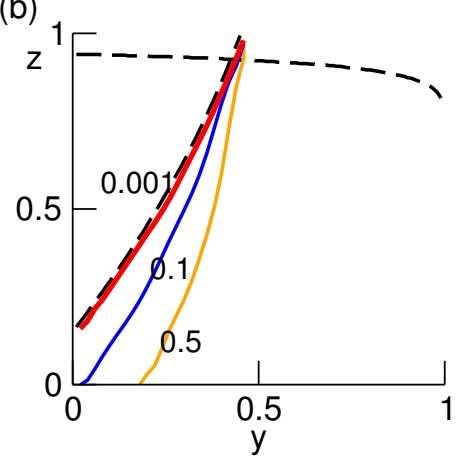

Figure 9. Contour lines of the probability of trajectories to reach a fixed point for different $\Delta t$. (a) $I=1$, (b) $I=0.85$. The solid lines give the initial locations where 90 of 100 trajectories approach the fixed point (a) $(1,1)(b)(1,0)$. Hence these lines are contour lines of the probability distribution to reach a stationary state with the fixed probability 0.9. The numbers in both panels are values of $\Delta t$ of the corresponding contour lines and the dashed lines represent the separatrix. Other parameters are taken from Fig. 6.

putation (Fig. 8(a)), the variables $y$ and $z$ are changed at the same time and thus the trajectory obeys the vectorfield $(\dot{y}, \dot{z})$, i.e. stays in the basin of attraction. Further in the shown example the vectorfield points to the fixed point FP1 and the length of the change vector $(\Delta y, \Delta z) \sim \Delta t$ does not point to locations outside the basin of attraction for both $\Delta t$. In contrast, the size of $\Delta t$ matters in the case of asynchronous computation (Fig. 8(b)). This evaluation type changes either $y$ or $z$ and thus the trajectory does not obey the vectorfield $(\dot{y}, \dot{z})$. Consequently it is possible that one variable changes in a way that the new trajectory point is located outside the basin of attraction. This probability to leave the basin of attraction is small for small $\Delta t$ since the length of the change vector is small, cf. (Fig. 8(b), line with circles. However larger $\Delta t$ yield a higher probability to leave the basin of attraction. As shown in Fig. 8(b), the trajectory might leave the basin and re-enter it.

In the previous paragraph we have discussed that trajectories computed synchronously are much less suceptible to $\Delta t$ than asynchronous trajectories since the latter does not obey the vectorfield $(\dot{y}, \dot{z})$ in each time step. To clarify this interplay between asynchronous evaluation and the size of $\Delta t$, Fig. 9 plots the initial locations of trajectories which approach the point $(1,1)$ (Fig. 9(a)) or $(1,0)$ (Fig. 9(b)) with the probability 0.9. We observe that the basin of attraction of the asynchronous trajectories depends on $\Delta t$ and increases with decreasing $\Delta t$. Further this asynchronous basin of attraction approaches the basin of attraction of the model (14), i.e. the synchronous basin of attraction. Consequently, the asynchronous computation is equivalent to synchronous computation for $\Delta t \rightarrow 0$.

\section{Conclusion and Future Directions}

This work distinguishes the synchronous and asynchronous evaluation scheme in dynamical systems and illustrates their different effects by numerical simulations in contiuum neural fields. To gain deeper insight into the phenomena observed, we introduce a low-dimensional model which exhibits similar behavior and allows to replace the CNFT-model in a first approximation. For this new model, the detailed analysis reveals the systems topology and uncovers subsequently the underlying differences of synchronous and asynchronous evaluation. At first, we observe that the system feels the presence of its fixed points for both evaluation schemes and hence obeys the systems topology. Consequently the system may approach its stable fixed points for both evaluation schemes. The only difference between the two 
schemes is the system trajectories, which do not necessarily obey the vector field of the dynamical system in the case of asynchronous evaluation and exhibits jumps in along a single coordinate axis due to its random nature. The strength of this random element in the asynchronous evaluation scheme depends strongly on the implementation time step. For very small time steps the random effects are reduced and the asynchronous evaluation resembles the synchronous evaluation. From the broader perspective of differential systems, we can make the conjecture that asynchronous evaluation with an infinitesimal $\Delta t$ is identical to synchronous evaluation with same $\Delta t$.

The results from the reduced model may give explanations for the behavior of the neural field dynamics using the asynchronous evaluation. For instance, according to neural field theory, i.e. theory of integral-differential equations, a single bump in neural fields does not exist in the presence of two bumps in the input, but may exist in numerical simulations applying the asynchronous computation scheme. The reason for this difference is the random nature of the asynchronous computation scheme, which allows the system to leave the basin of attraction of the stable fixed point representing two bumps and approach the stable fixed point representing a single bump, cf. Fig. 7 and 8. Moreover, the selection of the bumps in the asynchronous evaluation scheme is biased by the input as illustrated in Fig. 7: the element subjected to the stronger input is approached. This may explain the selection mechanism for both synchronous and asynchronous evaluation as observed in Fig. 3 and 4.

To learn more about the the neural field dynamics, we recall the relation of the nonlinear gain of the population firing rate function and the parameter $\alpha$ in the reduced model. The increase of $\alpha$, i.e. the increase of excitability, decreases the critical input $I_{c}$ (cf. section 4.1) and thus facilitates the preference of either $(1,0)$ or $(0,1)$ as the final state. In other words we argue that increasing the excitability in neural fields may improve the distinction of different input patterns and thus changes the visual attention. Indeed the relation of neural excitability and visual attention has been found experimentally [5, 24].

In realistic situations, one finds visual stimuli with different saliencies. Considering a neural population in the visual system and assuming an underlying asynchronous evaluation scheme, the visual system may choose the most salient stimulus and one may explain the stimulus selection by a stronger basin of attraction of the resulting pattern. In other words, the visual system may select the stronger bump with a higher probability than the other ones. However, the visual system may also select a bump with a lower saliency due to random nature of the systems trajectory, which however is much less probable (cf. Fig. 8).

Future work may study various model systems typically applied in computational neuroscience, such as a recurrent network of McCulloch-Pitts neurons, coupled FitzHugh-Nagumo or Hodgkin-Huxley models [11], or a network of spike-response neurons [17]. Especially the last model attracted much attention in the last years to analyse spiking neural networks. Even if such networks may benefit from a deterministic timing of spike emissions, they may be nonetheless considered in the light of asynchronous evaluation in their computational implementation.

\section{Acknowledgements}

The authors would like to thank Thierry Viéville and Frédéric Alexandre for valuable discussions. 


\section{References}

[1] S. Amari, Dynamic of pattern formation in lateral-inhibition type neural fields, Biological Cybernetics 27 (1977), pp. $77-88$.

[2] F.M. Atay and A. Hutt, Neural fields with distributed transmission speeds and constant feedback delays, SIAM J. Appl. Dyn. Syst. 5 (2006), pp. 670-698.

[3] D. Bertsekas and J. Tsitsiklis, Some aspects of parallel and distributed iterative algorithms - a survey, Automatica 27 (1991)

[4] D. Bertsekas and J. Tsitsiklis, Parallel and Distributed Computation: Numerical Methods, Athena Scientific, 1997.

[5] S. Bestmann, C. Ruff, C. Blakemore, J. Driver, and K. Thilo, Spatial attention changes excitability of human visual cortex to direct stimulation, Curr. Biol. 17 (2007).

[6] I. Bojak and D. Liley, Modeling the effects of anesthesia on the electroencephalogram, Phys. Rev. E 71 (2005), p. 041902

[7] P.C. Bressloff and S. Coombes, Physics of the extended neuron, Int. J. Mod. Phys. B 11 (1997), pp. $2343-2392$.

[8] A. Cadilhe, N. Araujo, and V. Privman, Random sequential adsorption: from continuum to lattice and pre-patterned substrates, J. Phys. Cond. Mat. 19 (2007), p. 065124.

[9] C.L.Barret and C.M.Reidys, Elements of a theory of computer simulation i: Sequential ca over random graphs, Applied Mathematics and Computation 98 (1999), p. 241.

[10] S. Coombes, Waves, bumps and patterns in neural field theories, Biol. Cybern. 93 (2005), pp. 91-108.

[11] P. Dayan and L. Abbott, Theoretical Neuroscience : Computational and Mathematical Modeling of Neural Systems, MIT Press, 2001.

[12] D.Frenkel and B.Smit, Understanding molecular simulation, Academic Press San Diego, 1996.

[13] G. Ermentrout and J. Cowan, A mathematical theory of visual hallucination patterns, Biol. Cybern. 34 (1979), pp. 137-150.

14] N. Fates, Asynchronism induces second order phase transitions in elementary cellular automata, Journal of Cellular Automata - (2008).

[15] S. Folias and P. Bressloff, Breathers in two-dimensional excitable neural media, Phys. Rev. Lett. 95 (2005), p. 208107.

[16] - Stimulus-locked waves and breathers in an excitatory neural network, SIAM J. Appl. Math 65 (2005), pp. 2067-2092.

[17] W. Gerstner and W. Kistler, Spiking Neuron Models, Cambridge University Press, Cambridge, 2002.

[18] X. Huang, W. Troy, S. Schiff, Q. Yang, H. Ma, C. Laing, and J. Wu, Spiral waves in disinhibited mammalian neocortex, J.Neurosc. 24 (2004), pp. 9897-9902.

[19] A. Hutt and F. Atay, Analysis of nonlocal neural fields for both general and gamma-distributed connectivities, Physica D 203 (2005), pp. 30-54.

[20] A. Hutt, M. Bestehorn, and T. Wennekers, Pattern formation in intracortical neuronal fields, Network: Comput. Neural Syst. 14 (2003), pp. 351-368.

[21] C. Laing and W. Troy, PDE methods for non-local models, SIAM J. Appl. Dyn. Syst. 2 (2003), pp. $487-516$.

[22] J. Lambert, Numerical methods for ordinary differential systems: the initial value problem, John Wiley and Sons, New York, 1991.

[23] L.D.Garcia, A.S.Jarrah, and R.Laubenbacher, Sequential dynamical systems over words, Applied Mathematics and Computation 174 (2006), pp. 500-510.

[24] V. Mountcastle, R. Andersen, and B. Motter, The influence of attentive fixation upon the excitability of the light- sensitive neurons of the posterior parietal cortex, J. Neurosci. 1 (1981).

[25] M.R. Owen, C.R. Laing, and S. Coombes, Bumps and rings in a two-dimensional neural field: splitting and rotational instabilities, New J.Phys. 9 (2007), p. 378.

[26] N. Rougier and J. Vitay, Emergence of attention within a neural population, Neural Networks 19 (2006), pp. 573-581.

[27] D. Steyn-Ross, M. Steyn-Ross, J. Sleigh, M. Wilson, I. Gillies, and J. Wright, he sleep cycle modelled as a cortical phase transition, J. Biol. Phys. 31 (2005), pp. 547-569.

[28] M. Steyn-Ross, D. Steyn-Ross, J. Sleigh, and L. Wilcocks, Toward a theory of the general-anestheticinduced phase transition of the cerebral cortex: I. a thermodynamic analogy, Phys. Rev. E 64 (2001), p. $011917 \mathrm{~J}$.

[29] J. Taylor, Neural bubble dynamics in two dimensions: foundations, Biological Cybernetics 80 (1999), pp. 5167-5174.

[30] N. Venkov, S. Coombes, and P. Matthews, Dynamic instabilities in scalar neural field equations with space-dependent delays, Physica D 232 (2007), pp. 1-15.

[31] T. Wennekers, Dynamic approximation of spatio-temporal receptive fields in nonlinear neural field models, Neural Comput. 14 (2002), pp. 1801-1825.

[32] W.H.Press, S.A.Teukolsky, W.T.Vetterling, and B.P.Flannery, Numerical Recipes, 3 Cambridge University Press, 2007.

[33] H. Wilson and J. Cowan, Excitatory and inhibitory interactions in localized populations of model, Biophysics 12 (1972), pp. 1-24.

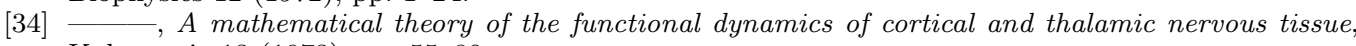
Kybernetic 13 (1973), pp. 55-80. 